\title{
XII.
}

\section{Pathologisch-anatomisclie Beobachtungen.}

\author{
Von Dr. M. Litten,
}

I. Assistenten an der med. Klinik u, Docenten an der Universität Berlin.

(Hierza Taf. VI - VII.)

\section{I. \\ Ueber einen Fall von infiltrirtem Leberkrebs nebst epikrilischen Bemerkungen.}

Die carcinomatösen Neubildungen in der Leber stellen sich bekanntlich in doppelter Form dar, einmal in Gestalt einzelner isolirter Knoten, welche gelegentlich zusammenfliessen und grössere Heerde bilden, alsdann aber in einer das ganze Organ ziemlich gleichmåssig betheiligenden Weise - als diffuse Infiltration. Während sich in diesem Organ bei ersterer Form häufig grosse, über das Niveau der übrigen Leber herausragende Knoten bilden, welche auf dem Durchschnitt sofort als solche zu erkennen sind, immer jedoch so, dass geschwulstfreies Parenchym zwischen den einzelnen Neubildungen übrig bleibt, es sei denn dass ein ganzer Lappen gelegentlich in die Neubildung aufgeht, präsentirt sich die diffus infiltrirte Leber in einer ganz anderen Form. Das gesammte Organ erleidet eine mehr oder weniger gleichmässige diffuse Krebsentartung, wobei dasselbe die denkbar grössten Dimensionen annehmen kann, immer jedoch unter Beibehaltung der früheren normalen Form. Ja selbst die acinöse Zeichnung kann trotz der carcinomatösen Degeneration so weit erhalten bleiben, dass die Erkrankung bei oberflächlicher Besichtjgung der Schnittfläche völlig übersehen werden kanı. Nach Schüppel, welcher dieser letzteren Form besondere Berücksichtigung schenkte, kommt dieselbe sowohl primär als secundär vor. Angesichts der eminenten Seltenheit des Auftretens secundärer Leberkrebse unter dem Bild der diffusen krebsigen Infiltration dürfte die folgende Mittheilung von allgemeinerem Interesse sein. 
Die Beobachtung betrifit eine 59jährige Fran, welche vom 5.-14. April 1879 ouf der medicinischen Klinik des Herrn Geh.-Rath Prof. Frerichs behandelt wurde. Anamnestisch wurde festgestellt, dass die Kranke bis zum Februar desselben Jahres ganz gesund war, alsdann aber eine Anschwellung des Lelbes bemerkte, welche alimäblich zunahm und im Lauf der nächsten Wochen bereits eine solche Höhe erreicht hatte, dass dadurch die Athmung behindert wurde. Sebr bald traten ausstrahlende Schmerzen auf, welche sich vom rechten Hypochondrium aus in's rechte Bein und nach dem Thorax bin erstreckten, und im März ein massiger lcterus, der beständig zunahm. Vorzugsweise dieser Schmerzen wegen suchte Pat, am 5. April die Charité auf.

Hier wurde bei der Aufnabme ein intensiver Ascites constatirt, welcher das Abdomen enorm ausgedehnt and das $Z$ werchfell beiderseits bis zum unteren Rand der IV. Bippe in die Höhe gedrängt batte. Trotz der Flüssigkeitsansammlung konnte man vermittelst kurzer energischer palpatorischer Stösse die Leber palpiren und namentlich im rechten Lappen ziemlich genau umschreiben. Dieselbe stellte sich dabei als ein sebr harter, grosser und auf der Oberfläche granulirter Tumor dar, welcher mit seinem unteren Rand quer durch das Epigastrium hindurch bis in's linke Hypochondrium verfolgt werden konnte, wo die Leberdämpfung unabgrenzbar in die vergrösserte Milzdämpfung überging. Schon spontan bestand eine sehr intensive Schmerzhaftigkeit der Leber, welche bei jeder Berührung zunahm. Pat. hielt deshalb beständig die Rückenlage ein und vermied so viel als thunlich jede stärkere Bewegung. Die Milz überragte die vordere Axillarlinie um $5-6 \mathrm{~cm}$. und bonnte unter dem Rippenbogen auf's Deutlichste gefühlt werden. Durch den Hochstand des Zwerchfells war in beiden unteren Lungenlappen eine Compression des Lungenparenchyms eingetreten. Sonst war in keinem inneren Organ eine Veränderung nachweisbar.

Die Haut icterisch, mit leichten Hämorrbagien durchsetzt. Die subcutanen Venen des Abdomens stark ectasirt und in grosser Ausdehnung als dicke blaue Stränge sichtbar. Fäces völlig entfărbt, Harn braungelb, frei von Eiweiss, zeigt bei Zusatz von unreiner Salpetersäure das charakteristische Farbenspiel.

Ueber den weiteren Verlauf der Krankheit ist za berichten, dass der Icterus und die Schwellung des Leibes, sowie die Schmerzhaftigkeit in der Lebergegend zunahmen. Von Seiten der Verdaung bestanden keine krankhaften Erscheinungen, namentlich war der Appetit beständig wohlerhalten. Der Harn, welcher anfangs meist icterisch gewesen war, nahm in den späteren Lebenstagen eine intensiv dunkelblaue Farbe an, welche zuweilen deutlich in's tief violette spielt. Abnorme Bestandtheile wie Eiweiss und Zucker wurden darin niemals nachgewiesen.

Am 14. April erfolgte der Tod, nachdem Pat. eine sebr gute Nacht verbracht und keine besonderen Klagen gehabt hatte.

Die Diagnose wurde auf Leberkrebs mit wahrscheinlicher Betheiliguag des Pfortadersystems (Compression? Verschluss durch krebsige Thromben?) gesteltt. Die Diagnose des Carcinoms stützte sich vorzugsweise auf die enorm rasche Eotwickelung des Leidens bei der sehr gut genährten Frau, auf die schnelle Entwickelung und rasche Zunahme des Icterus, sowie endlich anf die bedeutende Vergrösserung und enorme Schmerzhaftigkeit der Leber. Die unebene (granulirte) Beschaffenheit 
der Leberoberfläche wurde auf secundäre Mitbetheiligung des serösen Veberzuges bezogen. Ein Verschluss der grösseren Pfortaderäste endlich wurde angenommen wegen des grossen Milztumors und des bochgradigen Ascites.

Antopsie. Mittelgrosse, kräftig gebaute Leiche mit stark icterischer Hautfarbe. Auf den serösen Häuten finden sich viele bis linsengrosse, hämorrbagische Flecken. Herz und Lungen normal. Die Milz $19 \mathrm{Cm}$. lang, 12,5 Cm. breit, $5 \mathrm{~cm}$. dick; ihre Oberfläche mit zablreichen sclerotischen Platten besetzt. Der Magen ist an der kleinen Curvatur mit dem degenerirten Pancreas und den stark geschwollenen, epigastrischen Drüsen fest verwachsen. An der grossen Curvatur sitzen zwei längsovale, fast 10 Markstück-grosse, scharfrandige Geschwüre, deren Grund gereinigt ist und von der Muscularis des Magens, welche an ibrer eigenthümlichen streifigen Zeichnung leicht erkennbar ist, gebildet wird. Die Geschwürsränder sind ziemlich flach und frei von Knötchenbildungen. Daneben findet sich noch eine strablenfồrmige Narbe an der grossen Curvatur, doch näher nach dem Pylorus za. Der Magen, sowie das obere Drittheil des Dünndarms enthalten eine grosse Quantität dunklen, theerartigen Blutes. Die Schleimhaut des gesammten Digestionstractus erscheint nach der Abspülnng bis auf die vorerwähnten Ulcera ventriculi durchaus intact; auch an den letateren selbst findet sich keine Quelle der Blutung, namentjich keine sichtbare Arrosion eines Gefässes. Die gesammten Lymphdrüsen des Abdomens, die mesenterischen sowobl wie die retroperitonealen, namentlich aber die epigastrischen sind enorm geschwollen; die letzteren bilden ein männerfaustgrosses Packet, in welchem sich das ebenfalls total degenerirte, sehr harte und unförmliche Pancreas eingebettet findet. Die Leber ist in allen Durchmessern sehr stark vergrössert und sehr schwer (ca. 6,5 Kilo); an vielen Stellen finden slch strang- und flächenartige Synechien zwischen der serösen Bekleidung der Leber und dem Diaphragma. Die Oberfläche des Organs ist von intensiv gelber Farbe, uneben granulirt; die stark getrübte, stellenweise sehr bedeutend verdickte Serosa überzieht nicht nur die zahllosen Prominenzen nnd Höcker der Oberfläche, sondern steigt auch zwischen die Einziehungen derselben tief hinab. Auf der Schnittfläche erscheint das Organ eben, ziemlich gleichmässig gezeichnet und aur an ganz verejnzelten Stellen von kleinen grau-weisslichen Knötchen durchsetzt, welche als Neubildungen in's Parenchym eingesprengt sind; an einer Stelle findet sich ein grösserer weisslichgrauer rundlicher Knoten von dem Dmfang einer Haselnuss, welcher gegen das übrige Lebergewebe scharf abgesetat ist, im Centrum dellenartig vertieft und von einer stark hämorrhagischen Zone umgeben ist. Im Debrigen erkennt man eine Zeichnung, welche annähernd die normale Leberzeichnung wiedergiebt. Bei genauerer Betrachtung der Schnittfläche sieht man indess, dass diejenigen Partien, welche den Acinis entsprechen, sehr vergrössert, gelatinös-durchscheinend, ganz homogen und von grauer Farbe sind. Diese ,acinöse Zeichnung “ findet sich durch die ganze Drüse verbreitet, so jedoch, dass die als Acini erscheinenden Abschnitte tes Gewebes stellenweise von icterisch gefärbten Höfen umgeben sind, während sie

1 anderen Stellen von hämorrhagischen Zonen umfasst werden, welche bald einen - zelnen "Acinus", bald eine Gruppe derselben ziemlich regelmässig umschliessen. Der scheinbar acinöse Ban der Leber ist vielmehr durch diese Färbung, als durch die sichtbare Vertheilung der Blutgefässe bedingt. Durch diese Anordnung bekonmt 
die Leberfläche ein sebr - eigenthümliches und buntfarbiges, wie marmorirt erscheinendes Ansehen, welches die beiliegende Farbenzeichnung wiederzugeben versucht. Da, wo die icterischen Stellen vorherrschen, erscheint das Parenchym grau-gelb, wäbrend an den hämorrhagiscben Abschnitten ein mebr grau-rotber his intensiv rother Farbenton vorherrscht. Die grösseren Gallengänge und der Stamm der Pfortader sind frei. Die auf der Leberschaittfläche getroffenen und eröfloeten Aeste der lekzteren sind dagegen vielfach mit grau-röthlichen, markigen, ganz festhaftenden Thromben vollgestopft, deren konische Enden sich stellenweise in geronnene Gruormassen verlängern. In den übrigen Organen finden sich keine Veränderungen. Die Ven. pancreatica wurde leider nicht untersucht.

Liess die Section und namentlich das makroskopische Verhalten der Leber noch irgend einen Zweifel an der carcinomatösen Natur der Veränderungen, so musste derselbe bei der mikroskopischen Untersuchung vollständig schwinden. Die Veränderungen, welche sich bei letzterer fanden, waren so diffus durch das ganze Organ verbreitet, dass es nur der Herausnahme irgend eines beliebigen Stückes der Leber bedurfte, um dieselben überall und fast in der nehmlichen Ausdehnung wiederzufinden. Untersuchte man Querschnitte, welche aus irgend einem Abschnitt des Organs genommen waren (d. $h$. Schnitte, welche die Imenvene senkrecht zu ihrer Axe trafen), so erkannte man bei schwacher Vergrösserung noch an den meisten Stellen ten groben Bau der Leber, die acinőse Anordnung sowie den Verlauf der inter- und intralobulären Venen. Die einzelnen "Acini" scheinen dabei nicht unerheblich vergrössert. Bei Anwendung stärkerer Vergrösserungen jedoch bemerkt man sofort, dass die interlobulären Gallengänge vielfach versehwunden, die Leberzellenbalken durch abnorm breite $Z$ wischenräume von einander getrennt und selbst auf's Aeusserste verschmälert sind. Die einzelnen Leberzellen sind entsprechend dieser Verschmälerung der Zellenbalken ausserordentlich comprimirt und atrophisch, dabei aber durch ihre gelbliche Farbe, granulirte Beschaffenheit und ihren reichlichen Gehalt an Gallenpigment leicht als solche zu erkennen. Die Verschmälerung der Leberzellenbalken hatte, wie leicht nachweisbar war, ihren Grund in einer z. Th. enormen Ausdehuung der dazwischen gelegenen Capillaren, welche sich auf Kosten jener in colossalem Maasse erweitert hatten. Zum Theil waren die Leberzellenbalken so comprimirt, dass sie die eetasirten Capillaren, als ein weitmaschiges Netzwerk, dessen Maschenräume mit eigenthümlichen zelligen Bildungen ausgefüllt waren, umgaben. Die bis zu 
einer Weite von $60 \mu$ und stellenweise weit darüber ausgedehnten Capillargefässlücken ${ }^{1}$ ) enthielten zum grossen Theil noch wohlerhaltene rothe Blutkörperchen und daneben ausnahmslos sehr grosse rundliche oder polygonale, granulirt aussehende Zellen (durchschnittlich $15-20 \mu$ ) mit sehr grossem stark lichtbrechendem Kern und Kernkörperchen. Diese Zellen selbst, welche zuweilen birnförmig erschienen, waren mit sehr feinkörnigem Inhalt versehen und von äusserst mattem Glanze. Nirgends fand sich in ihnen eine Spur von Pigment. Während trotz dieser enormen Ectasie der Capillaren und der dadurch hervorgebrachten Compression der Leberzellenbalken die gröbere Structur des Organs noch erhalten erscheint, ist dieselbe in den vorhin beschriebenen miliaren Knötchen und den ganz vereinzelten grösseren Knoten gänzlich zu Grunde gegangen. In diesen zuletzt erwăhnten partien erkennt man ohne Weiteres den alveolären Bau des Carcinoms, wobei die einzelnen Alveolen in einem äusserst zarten Stroma eingebettet sind, welches bin und wieder einen gelblichen Farbenton hat und den Eindruck hervorruft, als ob es aus den Resten der gänzlich zu Grunde gegangenen Leberzellenbalken bestünde. In dem die Interlobulargefässe begleitenden Bindegewebe findet man, soweit die Neubildung diffus ist, einen etwas vermehrten Gehalt-an Rundzellen, welche aber nirgends grössere Heerde bilden. In den kleinsten Pfortaderästchen fanden sich ebenso wie in den Pfröpfen, welche stellenweise in den grösseren Aesten desselben Gefässes steckten und makroskopisch erkennbar waren, die nehmlichen grossen Zellen mit grossem glänzenden Kern, welche wir überall im Innern der erweiterten Capillaren angetroffen hatten.

Der mikroskopische Befund konnte demnach darüber keinen Zweifel lassen, dass im vorliegenden Fall eine diffuse, fast ganz gleichmässige Infiltration der Leber mit Krebszellen bestand, d. h. ein infiltrirter Leberkrebs, wobei jeder Leberacinus durch eine Anzahl von Krebsacinis substituirt war. Die Wandungen der ectasirten Capillaren bildeten dabei mit den Residuen der comprimirten und erdrückten Leberzellenbalken ein Gerüstwerk mit kleinen, ziemlich regelmässigen Alveolen, in welchen sich genau die nehmlichen zelligen Elemente wiederfanden, welche der Hauptmasse nacb

t) Der normale Durchmesser dieser Capiliaren beträgt bei mässiger Füllung ungefähr $10 \mu$. 
jene embolischen Pfröpfe bildeten, welche, wie die Abbildung zeigt, aus den Durchschnitten der Pfortaderäste herausragten. Jeder Zweifel an der Richtigkeit dieser Auffassung muss schwinden, wenn wir die genetische Beziehung des Falles ins Auge fassen. Die Kranke litt an einem primären Carcinom des Pancreas, zu welchem eine secundäre Leberaffection binzugetreten war. Nimmt man dazu die aus grossen Krebszellen bestehenden embolischen Pfröpfe der Pfortader, in welchen man auf Schnitten stellenweise sogar den völligen Charakter des Carcinoms mit Stroma und Alveolen auf's Deutlichste nachweisen komnte, sowie das Vorhandensein der nehmlichen Gebilde in den zwischen Pfortader und Ven. hep. eingeschalteten Capillaren, so wird man trotz der fehlenden Untersuchung der pancreatischen Venen und des Nachweises, dass der Krebs in die Venen der Bauchspeicheldrüse hineingewuchert war, doch zu dem Schluss gezwungen, dass embolisches Material aus dem entarteten Pancreas durch die Venen in die Pfortaderåste hineingeschwemmt wurde, zum Theil an Ort und Stelle weiter wucherte, zum Theil aber in kleinste Partikel zerbröckelt und in die Capillaren hineingetrieben wurde. Hier bildete das embolische Material die Seminien für die Ausdehnung der Neubildung, welche vorzugsweise a u die Capillaren beschränkt blieb und nur da zu grösseren Knoten confluirte, wo durch die besonders starke Ausdehnung der Capillaren das Lebergewebe völlig erdrückt wurde. Hierfür sprachen auf's Unzweideutigste diejenigen Bilder, welche aus Leberabschnitten stammten, die den Uebergang der diffusen Infiltration in die Krebsknoten erkennen liessen. Das eigentliche Lebergewebe, $d . h$. die Leberzellen, betheiligten sich also an dem Prozess selbständig in keiner Weise, wurden vielmehr nur seeundär durch den Druck der wachsenden Neubildung resp. der ectasirten Capillaren in Mitleidenschaft gezogen. Dass die Neubildung ihren Sitz wirklich ausschliesslich in den Capillaren hatte, geht daraus hervor, dass die Krebszellen überall gleichzeitig mit rothen Blutkörperchen zusammen angetroffen wurden und zwar in präformirten Hohlräumen (Lücken), an deren Wand man vielfach endotheliale Zellen erkeunen konnte. An denjenigen Stellen, wo innerhalb der Capillaren nur wenige Krebszellen lagen, eine sehr erhebliche Ausdehnung derselben mithin noch nicht erfolgt war, sah man die Balkenreihen der Leberzellen relativ noch sehr wohl erhalten; in demselben Maasse jedoch, in 
welchem die Neubildung und dadurch auch die Ausdehnung der Capillaren zugenommen hatte, zeigte sich eine immer hochgradigere Compression des Lebergewebes, bis sich schliesslich die Wandungen der nunmehr übermässig erweiterten Capillaren fast berührten, so dass schliesslich die präexistirenden Gefäss(Capillar) räume die Krebsalveolen, die Capillarwände plus der Reste der geschwundenen Lebersubstanz, welche sich in den extremsten Fällen als eine schmale, körnig trübe, gelbliche Substanz darstellte, die Alveolarwände (i. e. das Krebsstroma) darstellten.

Wir sehen in der Art, wie sich die Neubildung im vorliegenden Fall von dem Pancreas auf die Leber propagirte, das vollkommenste und typischste Beispiel einer Implantation des Krebses im Sinne von Thiersch, wobei Krebszellen aus der primären Geschwulst in ein anderes Organ implantirt, und hier zur Ursache der Geschwulstmetastasen werden. Ob diese Krebszellen, welche in die Gefässbahn des secundären Organs einwandern, hier einfach als harmloses embolisches Material mechanisch wirken, oder zur Weiterentwicklung gelangen und dadurch zur Metastasirung des Neoplasma beitragen, hängt wohl zweifellos von individuellen Eigenthümlichkeiten, Disposition, hereditären Einflüssen etc. $a b, d . h$. von uns unbekannten Factoren, die aber in Wirklichkeit sicher rorhanden sind. Ich verweise in dieser Beziehung auf die Auseinandersetzungen Cohnheim's in dessen Allg. Pathologie, S. 668 u. ff. Bekanntlich ist es dem genannten Autor und Maas gelungen, legitimen Knochen in den Pulmonalarterienästen von Hühnern zu erzeugen, welchen lebenswarmes Periost in die Jugularvenen eingebracht wurde. Allerdings verschwanden die neugebildeten Knochenplatten innerhalb der nächsten Wochen, und zwar weil, wie Cohnheim ausführt, die fremden Partikel dem Stoffwechsel der physiologischen Gewebe nicht zu widerstehen vermögen. Wenn andererseits Gewebe das Eindringen von Geschwulstzellen sowie die Weiterentwicklung und das Wachsthum von eingeschwemmten Geschwulstpartikeln gestatte, so schliesst Cohnheim weiter, könne sich dasselbe nicht mehr physiologisch verhalten. Der Verlust oder die Abnahme dieser physiologischen Widerstandsfähigkeit der Gewebe ist theils in localen, theils in allgemeinen Ursachen zu suchen. Von letzteren dürften für die "krebsige Diathese“ vorzugsweise das Alter des Individuums, die Erblichkeitsverhältnisse sowie die Prä- 
disposition (das Vorhandensein von Carcinomen in anderen Organen) etc. heranzuziehen sein. Im vorliegenden Fall handelt es sich um eine ältliche Frau mit primärem Pancreaskrebs und nachweisbarer hereditärer Belastung, da die Mutter der Patientin einer Neubildung wegen an der Brustdrüse operirt worden war.

Dass unsere Auffassung von der e $m b$ olis ch en Verbreitung des Krebses im mitgetheilten Fall eine richtige ist, und dass es sich hierbei nicht um directe Fortpflanzung des Carcinoms vom Pancreas aus auf die Leber per continuitatem mit secundärem Durchbruch in die Pfortader gehandelt habe, geht daraus hervor, dass die Porta hepatis und der Stamm der Pfortader nebst deren grösseren Aesten vollständig frei von der Neubildung gefunden wurde. Nirgends, soweit unsere Untersuchung reichte, fanden wir auf den vielfachen Durchschnitten, welche wir durch die Leber machten, irgend einen Ast der Vena port. von der Neubildung durchwuchert; überall war die Gefässwand völlig intact und nur im Lumen des durchschnittenen Gefässes fand sich ein Krebsembolus, welcher an seinen Enden noch Blutgerinnsel erkennen liess, ein Beweis, dass es sich nicht um absoluten Verschluss der betreffenden Aeste mit völliger Unterbrechung der Circulation gehandelt habe. Und selbst wenn dieser Modus der Durchwucherung eines oder des anderen Astes der Pfortader mit Krebsmassen an irgend welchen unentdeckt gebliebenen Stellen stattgefunden hätte, so würde dieser Vorgang noch keineswegs eine so gleichmässige Erfüllung sämmtlicher Capillaren mit Krebszellen erklären; denn kein Stück der Leber, von welcher Stelle es auch genommen wurde, war frei von den beschriebenen Veränderungen. Indess war die Infarcirung der Capillaren mit den Krebszellen keine so vollständige, dass dadurch die capilläre Circulation aller Orten ernstlich gelitten hätte, denn einmal fanden sich an vielen Stellen des Capillarsystems neben den krebsigen Embolis noch die körperlichen Bestandiheile des Blutes in völlig erhaltenem Zustande, und ferner zeigte die Beschaffenheit des Organs auf's Unzweideutigste, dass die Ernährung desselben im Grossen und Ganzen nicht erheblich gelitten haben konnte. Allerdings war stellenweise eine starke Hyperämie gewisser grösserer Leberabschnitte vorhanden, welche darauf hinwies, dass der Abfluss des Pfortaderblates beeinträchtigt wäre, doch war es innerhalb der Leber nirgends zu Hämorrhagien oder blutigen Infarcirungen gekommen. Ebensowenig 
aber fanden sich ausser der Druckatrophie der Leberzellen Ernährungsstörungen regressiver Art in der Leber vor, welche auf eine wesentliche Beeinträchtigung der Ernährung hätten schliessen lassen. Es kann dies letztere um so weniger Wunder nehmen, da das für die Ernährung der Leber wichtigste Gefäss - die Art. hepatica völlig frei und durchgängig geblieben war. Dadurch wird auch die enorme Grössenzunahme des Organs trotz der Verlegung vieler Pfortaderäste verständlich. Dazu kommt ferner der Umstand, dass die interlobulären Aeste der Vena port. überall frei geblieben waren, so dass selbst diejenigen interlobulären Venen, deren zuführende Pfortaderäste verstopft waren, noch immer arterielles Blut erhalten konnten. Bekanntlich ergiessen die Leberarterien ihr Blut zum grössten Theil in diese interlobulären Venen, so dass eine genügende Ernährung des Organs selbst nach Pfortaderverschluss (Pylephlebitis etc.) noch stattfinden $k^{2}{ }^{3}$ ).

Die Erklärung des Icterus dürfte auf grosse Schwierigkeiten stossen, da die anatomische Untersuchung keine genügende Ursache für denselben nachwies. Die Gallenausführungsgänge sowie die grösseren Gallengänge innerbalb der Leber waren frei, nur waren die interlobulären Gallengänge vielfach geschwunden. Nimmt man hierzu die Thatsache, dass die im Innern der Leberinseln gelegenen Gallencapillaren comprimirt und beeinträchtigt waren, so würde dies immerhin einen Anhaltspunkt für das Zustandekommen des Icterus abgeben. Dagegen ist jedoch zu bemerken, dass die Leberzellen derjenigen Acini, in welchen die Ausdehnung der Blutcapillaren und dadurch bedingt die Compression der Gallencapillaren ihre grösste Höhe erreicht hatte, gerade am meisten gelitten hatten und sicherlich keine Galle mehr bildeten. Andererseits aber kann man den intensiven Icterus, der intra vitam bestanden hatte, auch nicht zurückführen auf den Untergang der Leberzellen, da immerhin noch eine ganz beträchtliche Anzahl der letzteren in ihrer Form völlig erhalten war und normal functionirte, wie die Galle bewies, welche wir in der Gallenblase vorfanden, und namentlich auch aus dem Umstand mit absoluter Sicherheit hervorgeht, dass trotz des intensiven Icterus niemals eine Decolorisation der Fäces bestand. Ausserdem waren während des Lebens niemals die Symptome der sogenannten "Acholie“ vorhanden gewesen.

') Vergl, über liese Verbältnisse: Cohnheim-Litten, dieses Archiv Bd. 67. 
Der mitgetheilte Fall gehört seinem anatomischen Verhalten nach zu den allerseltensten Vorkommnissen, deren in der Literatur bisher nur von sehr wenigen Autoren gedacht worden ist. Den ersten dieser seltenen Fälle von diffuser intracapillärer Infiltration der Leber mit Krebsmassen beschreibt, soweit ich sehe, Schüppel (Archiy der Heilkunde Bd.9.). Dieselbe nahm im genannten Fall ihren Ausgang von einer Neubildung der Chorioidea, welche später nach der Milz metastasirte und von hier aus zu einer Transplantation der Krebszellen nach den Lebergefässen führte; demgemäss handelte es sich um ein melanotisches Carcinom. Aber abgesehen von diesem letzteren, ganz indifferenten Unterschiede, ähnt mein Fall dem von Schüppel in erschöpfender Weise beschriebenen so vollständig, dass sie einander fast decken. Später beobachtete Schiippel eine ähnliche Verbreitung der Neubildung in einem Fall von Gallertkrebs der Leber (cf. Ziemssen's Sammelwerk. VIII. 1. S. $293 \mathrm{ff}$.). In ähnlicher Weise scheint sich namentlich nach Rindfleisch's Angaben auch der Strahlenkrebs oder das Radiärsarcom der Leber zu verbreiten. Auch Perls sah eine Verbreitung und Fortpflanzung des Carcinoms durch die Capillaren sowohl in der Chorioidea wie in der Leber (cf. dies. Arch. Bd. 56.). - Viel häufiger kommt es vor, dass von einem carcinomatösen Heerd aus ein Durchbruch in den Stamm oder einen grösseren Ast der Vena port. erfolgt, und dass von hier aus die carcinomatösen Massen dem Blutstrom folgend oder auch in entgegengesetzter Richtung (cf. Späth, dies. Arch. Bd. 35) fortwuchern und zur Verbreitung des Neoplasma beitragen. Auf diesem Wege kommt es zur Metastasenbildung vereinzelter Knoten, aber nicht zu der eben beschriebenen diffusen Infiltration der Capillaren. Letztere kommt übrigens, wie Waldeyer gezeigt hat (dies. Arch. Bd. 41.) auch in den Capillaren der Lymphgefässe zur Beobachtung.

Abgesehen von der ungewöhnlichen Verbreitung der Neubildung scheint mir der vorliegende Fall noch nach anderen Richtungen hin Bemerkenswerthes darzubieten:

1) Das gleichzeitige Vorkommen runder Magengeschwüre neben Carcinom der Verdauungsdrusen (Panereas und Leber). Als bei der Autopsie die beiden Geschwüre, welche an der grossen Curvatur ihren Sitz hatten, gefunden wurden, glaubte man den Ausgangspunkt der gesammten Erkrankung im Magen suchen zu müssen. 
Sprach indess schon die strahlige Narbe, welche gleichfalls an der grossen Curvatur sass, gegen die carcinomatöse Natur der anderen Ulcera ventriculi, so verlor diese Anschauung vollends ihren Halt, als durch die mikroskopische Untersuchung der Nachweis geliefert wurde, dass jede Spur einer krebsigen Neubildung am Grunde oder den Rändern der Magengeschwüre fehlte. Mithin ist die krebsige Entartung des Pancreas als das Primäre, die der Leber als das Secundäre aufzufassen, während die Affection des Magens als eine davon unabhängige Complication zu betrachten ist.

2) Die Staungen im Gebiet der Pfortader, welche sich bis zur tödtlichen Hämorrhagie steigerten. - Sämmtliche zum System der Ven. port. gehörigen Organe waren durch die Verlegung eines grossen Theils der intrahepatischen Pfortaderausbreitung in Mitleidenschaft gezogen worden. Dies äusserte sich während des Lebens nachweisbar in einer bedeutenden Schwellung der Milz und dem schnell wachsenden umfangreichen Ascites. Die Autopsie machte es ferner mehr als wahrscheinlich, dass die den Tod bedingende Gastroenterorrhagie ebenfalls mit den Stauungen im Stromgebiet der Ven. port. im engsten Zusammenhang stünde. Wie ich schon gelegentlich der Mittheilung des Sectionsbefundes hervorhob, konnte man nirgends an den Magengeschwüren eine Ursache für die Blutung auffinden; nirgends fand sich daselbst eine Gefässarrosion, ein Aneurysma oder auch selbst nur ein festanhaftendes Blutgerinnsel. Die Möglichkeit, dass es sich um eine Stauungsblutung gehandelt habe, liegt zweifellos vor, da man Aehnliches bei der Lebercirrhose und der Verlegung der Pfortader antrifit. So erwähnt Frerichs einen ähnlichen Fall von tödtlicher Magendarmblutung bei Obliteration der Ven. port., bei welchem sich nirgends Erosionen oder Ulcerationen der Darm- oder Magenschleimhaut vorfanden (Klinik der Leberkrankh. Bd. I. S. 280). Ich selbst beobachtete eine tödtliche, scheinbar aus dem Magen stammende Blutung bei einem an Cirrhosis hepatis leidenden Individuum, bei welchem die Section keine andere Quelle der Hämorrhagie nachwies, als enorme submucöse Varicen im untersten Theil des Oesophagus, die jedoch an keiner Stelle ulcerirt waren.

3) Das Auftreten eines tief dunkelbau-violetten Harns, welcher diese Farbe bereits unmittelbar nach der Entleerung darbot. Es kann keinem Zweifel unterliegen, dass die Blaufärbung zum gröss- 
ten Theil auf dem Vorhandensein von Indican beruhte, welches z. Th. bereits gespalten war. Vielleicht concurrirte dabei auch das Auftreten von Oxydationsproducten des Phenols (Hydrochinon und Brenzkatechin). Bekanntlich hat man bei Magen- und auch bei Lebercarcinomen sowohl eine starke Ausscheidung von Indican wie von Phenol (Brieger) durch den Harn beobachtet. Indess scheint nicht das gesammte Phenol als solches ausgeschieden $\mathrm{zu}$ werden, sondern z. Th, als 0xydationsproducte desselben (Hydrochinon und Brenzkatechin), wie Baumann und preusse gezeigt haben.

Wiederholt hat man im Harn von Individuen, welche an Pigmentkrebs litten, das Vorhandensein eines Farbstoffes nachgewiesen, welcher durch Oxydation beim Stehen an der Luft oder auf Zusatz von Salpetersäure schwarz wird. Leichtenstern erwähnt auch eines Falles von (nicht pigmentirtem) Krebs des Magens, bei welchem auffallend dunkler, schwarzbrauner Harn entleert wurde, welcher auf $\mathrm{HNO}_{3}$-Zusatz ganz schwarz wurde (cf. Ziemssen's Sammelwerk. VIll. 1. S. 343). Ich selbst habe mehrere Male bei nicht melanolischem Leberkrebs das Auftreten eines dunkelviolett-blauen Harns beobachtet, welcher bereits unmittelbar nach der Entleerung diese Farbe zeigte. Nach langem Stehen an der Luft trat eine wesentliche Farbenveränderung ein, indem sich der violette Farbenton in einen tiefgrauen bis schwärzlichen umwandelte. Stets konnte ich dabei einen hohen Indicangehalt nachweisen. In einem Fall von gleichzeitig bestehendem intensiven Icterus konnte im Harn das Vorhandensein von Gallenfarbstoff nicht constatirt werden.

4) Die gleichzeitige Erkrankung so vieler Digestionsorgane ohne jede Spur einer Verdauungsstörung während des Lebens. Trotz der totalen Pancreasdegeneration, der weit verbreiteten Compression des secernirenden Lebergewebes durch das Neoplasma, ja selbst trotz des Vorhandenseins mehrerer Magengeschwüre fehlte jedes Symptom von Seiten des Digestionsapparates, so dass sowohl die Ulcera ventriculi als die Entartung des Pancreas während des Lebens nicht erkannt worden waren. Derartigen Fällen begegnet man nicht so ganz selten, und namentlich kann eine weit verbreitete Erkrankung, ja selbst totale Degeneration des Pancreas bestehen, ohne dass irgend eines der für Pancreaserkrankungen als charakteristisch angegebenen Symptome (Fettstühle, Lipurie, Broncehaut, Neuralgia coeliaca, Mellit- 
urie, Salivatio pancreatica etc.) vorhanden wäre. Eine Reihe derartiger, klinisch genau beobachteter Fälle habe ich in den beiden letzten Bänden der Charité-Annalen (Bd. IV und V) beschrieben, wo der hier mitgetheilte Fall ebenfalls Berücksichtigung gefunden hat.

\title{
II. \\ Ueber embolische Muskelveränderung und die Resorption codter Muskelfaseru.
}

\author{
Ein Beitrag zur Frage von der Ueberwanderang embolischen \\ Materials bei offen gebliebenem Foramen ovale.
}

Pauline Schütz, 43 Jabre alt, aufgenommen auf die medicinische Klinik des Herrn Geb.-R. Frerichs am 20. Februar 1878, gestorhen am 8. März dess. J.

Die ersten Klagen der Pat. datiren aus dem Sommer 1877, um welche Zeit starker Husten mit Auswurf aufgetreten sein soll. Derselbe bestand trotz ärztlicher Behandlung nicht nur fort, sondern nahm im Lauf der nächsten Monate an Intensität bedeutend zu und fübrte zu starker Entkräftung und Abrnagerung. In den ersten Tagen des nächsten Jabres soll ein einmaliger Schültelfrost aufgetreten sein, welchem bald neue Erscheinungen von Seiten der Brustorgane nachfolgten. Es traten heftige Schmerzen in beiden Seiten des Thorax auf, welche beim Athmen stärker wurden, ausserdem wurden gleichzeitig zum ersten Mal während der Krankheit blutige Sputa expectorirt. Die Athemnoth nabm bedeutend zu. Diese Affection ging zwar nach einigen Tagen vorüber, alsbald aber traten nach kurzen Prodromalerscheinungen von perversen Empfindungen im rechten Bein heftige Schmerzen in derselben Extremităt auf, welehe nach einigen Tagen in völlige Empfindungslosigkeit äbergingen. Gleichzeitig bemerkte Pat. eine Beeinträchtigung der Functionsfähigkeit dieses Beines, welche sich binnen Kurzem bis zur völligen Unfähigkeit, die Extremität zn gebrauchen, steigerte. Anfangs bestand auch eine Schwellung der Extremität, welche sich nach einiger Zeit verlor. Drei Wochen nach Begino der Gesammtaffection bemerkte die Kranke, dass die Haut des rechten Fusses zusanmenschrumpfte und eigenthümlich runzlig wurde; einige Zeit darauf verfärbte sich die Haut des gesammten Unterschenkels und uahm einen grünlich-grauen Farbenton an. Trołz des Bestehens yon Anästhesie und Analgesie in der nuumehr völlig ausser Function gesetzten Extremität sollen spontan häufig Anfälle von dumpfem Schmerzgefühl darin aufgetreten sein. Da gleicbzeitig Husten und Auswurf intensiver wurden, suchte Pat. am 20. Februar die Klinik anf, wo folgender Status aufgenommen wurde:

Die sebr dürftig genährte, stark abgenagerte Kranke bietet bei der äusseren Besichtigung sebr intensive Veränderungen des rechten Unterschenkels dar. Die Haut des letzteren, welche in der gesommten Ausdebnung vom Knie an bis zum Fuss grau-grünlich verfärbt, stellenweise von stahlgrauem oder graphitartigem Farbenton ist, erscheint überall gernnzelt und gefaltet, als ob sie für die Unterlage viel zu weit sei. Dicht unterbalb des Kniegelenks beginnt eine bis etwa 2 fingerbreit 


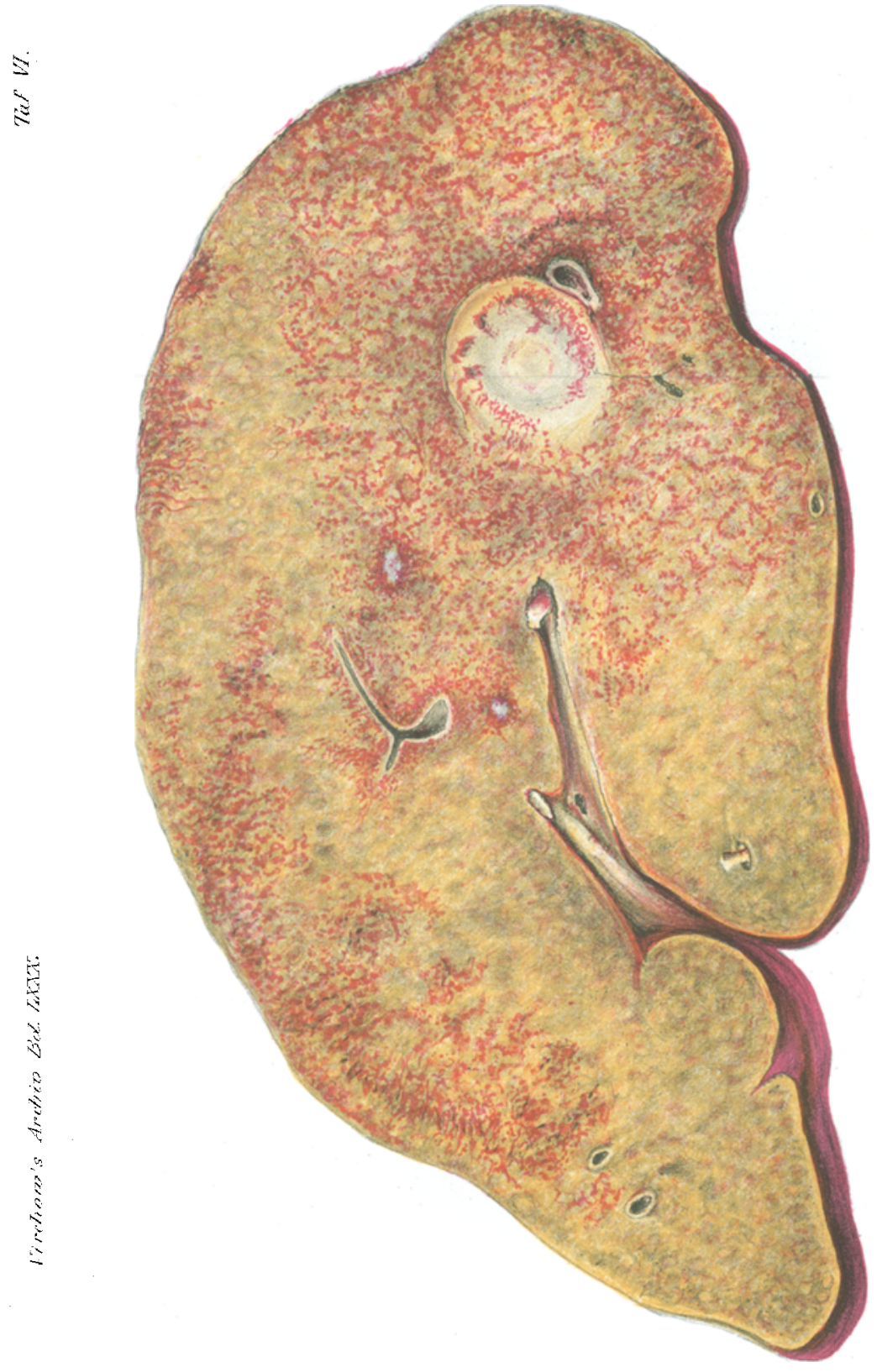



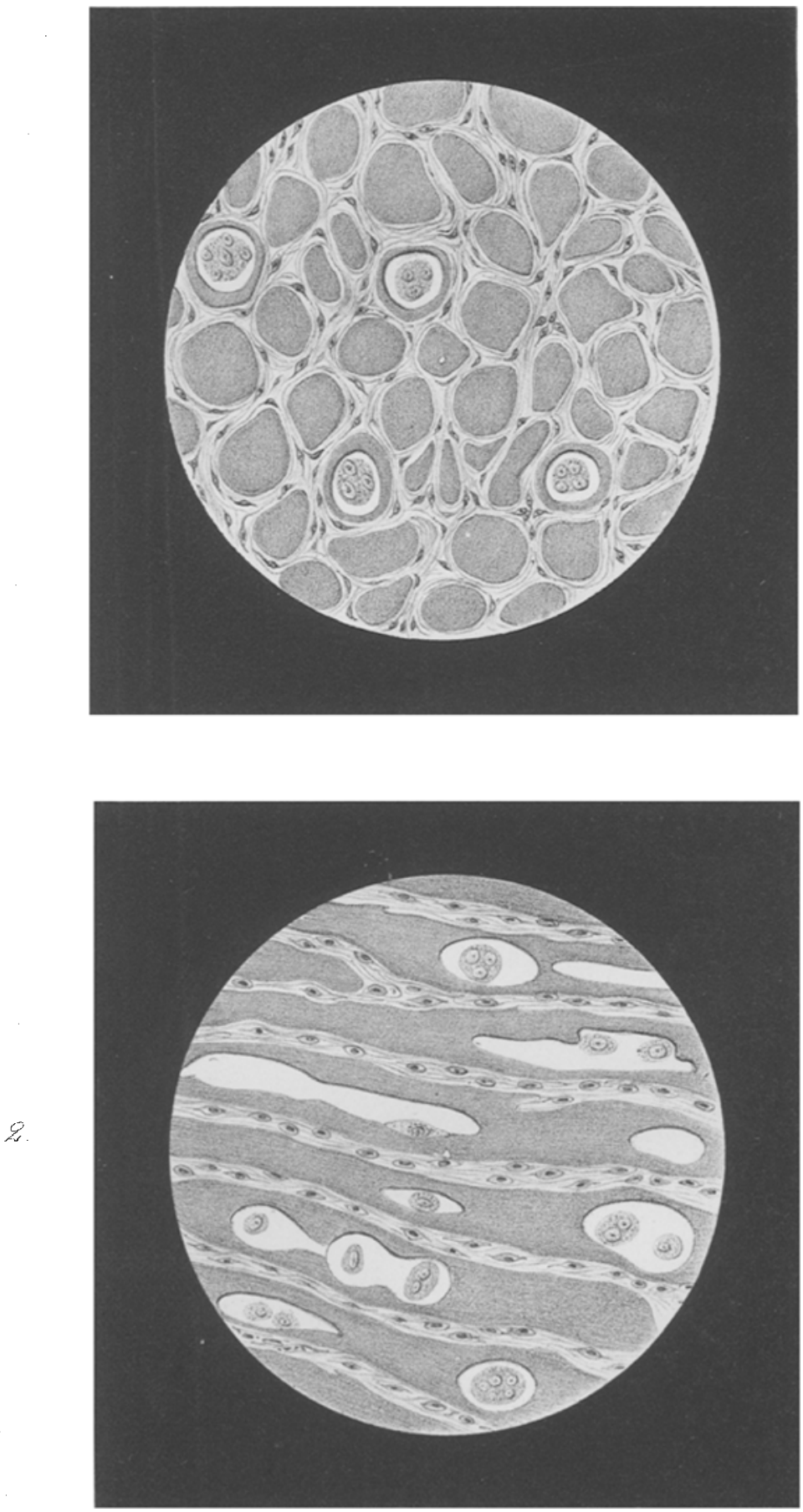